den und einem anderen Staat zurückzugeben ist. Es besteht im Übrigen keinerlei Anhaltspunkt dafür, dass der Kläger nicht Eigentümer der rechtswidrig sichergestellten Münzen geworden ist.

Die Sicherstellung kann auch nicht auf $\S 40$ Nr. 4 HSOG gestützt werden, denn unabhängig davon, dass $\S 8$ Abs. 6 KultGüRückG die speziellere Norm ist, liegen auch, wie das Verwaltungsgericht zutreffend ausführt, keine Anhaltspunkte dafür vor, dass die Münzen zur Begehung einer Straftat oder einer Ordnungswidrigkeit verwendet werden sollen.

Der Zulassungsgrund der besonderen tatsächlichen und rechtlichen Schwierigkeiten der Rechtssache (§ 124 Abs. 2 Nr. 2 VwGO) erfordert überdurchschnittliche, das normale Maß nicht unerheblich überschreitende Schwierigkeiten (vgl. dazu Kopp/ Schenke VwGO, 15. Aufl. 2007, § 124, Rn. 9). Zu überprüfen war die Frage, ob die Beklagte die Sicherstellung der Münzen auf der Grundlage des $\S 40$ Nr. 2 und 4 HSOG vornehmen durfte. Wie die Ausführungen im Rahmen der Prüfung des § 124 Abs. 2 Nr.1 VwGO ergeben haben, lässt sich diese Frage anhand des Gesetzes beantworten und wirft keine überdurchschnittlichen Schwierigkeiten auf.
Auch der Zulassungsgrund der grundsätzlichen Bedeutunq der Rechtssache im Sinne des $§ 124$ Abs. 2 Nr. 3 VwGO ist nicht hinreichend dargetan. Zur Darlegung dieses Zulassungsgrundes ist es erforderlich, eine bestimmte, bisher noch ungeklärte und für die Entscheidung des Oberverwaltungsgerichts/Verwaltungsgerichtshofs erhebliche Rechtsfrage zu formulieren und anzugeben, worin die allgemeine, über den Einzelfall hinausgehende Bedeutung dieser Rechtsfrage bestehen soll (vgl. Hess. VGH Beschluss vom 17. Juli 1998 - 8 ZU 2071/98 -, unter Hinweis auf BVerwG Beschluss vom 19. August 1997 - 7 B 261/97 = NJW 1997, 3328). Eine solche Darlegung lässt sich jedoch den Ausführungen des Beklagten nicht entnehmen. Die von dem Beklagten formulierte Frage lässt sich anhand der gesetzlichen Vorschriften beantworten.

Die Kostenentscheidung beruht auf § 154 Abs. 2 VwGO, die Streitwertfestsetzung folgt der erstinstanzlichen Wertfestsetzung, zumal der Beklagte dagegen keine Einwendungen erhoben hat.

Dieser Beschluss ist unanfechtbar (§ 152 Abs. 1 VwGO: $\S 66$ Abs. 3 Satz 3 GKG iVm $\S 68$ Abs. 1 Satz 5 GKG). (Entscheidung von der Redaktion bearbeitet.)

\title{
Zum Verhältnis von Anhaltungsverfügung nach KultGüRückG und polizei- rechtlicher Sicherstellungsverfügung (II)
}

VG Gießen, Urteil vom 6. Mai 2010 - 9 K 1661/09.GI

\section{1. § 8 Abs. 1, 6 KultGüRückG geht aufgrund der besonderen Ausformung des Eigentumsschutzes als lex specialis} der Vorschrift des $\$ 40$ Nr. 2 HSOG vor.

2. Nach § 85 Abs. 1 Nr. 1 HSOG können die fachlich zuständigen Ministerien zwar als Landesordnungsbehörden und damit als allgemeine Ordnungsbehörden handeln; dies jedoch nur bei einem Handeln im Rahmen der fachlichen Zuständigkeit, die sich indes über die Vorschriften des Kulturgüterrückgabegesetzes definiert und für die Sicherstellung von Kulturgütern die Spezialregelung des § 8 Abs. 1, 6 KultGüRückG vorsieht; eine darüber hinausgehende Zuständigkeit zur präventiven Bekämpfung von Straftaten und Ordnungswidrigkeiten durch Sicherstellungsmaßnahmen gemäß $\$ 40$ Nr. 4 HSOG ist nicht eröffnet. Darüber hinaus zählt der Bereich des Kulturgüterschutzes nach dem Kulturgüterrückgabegesetz nicht zu den, den allgemeinen Ordnungsbehörden gemäß § 1 HSOG-DVO, § 89 Abs. 1 HSOG zugewiesenen Aufgabenbereichen.

3. Die Umdeutung einer angefochtenen Sicherungsverfügung in eine entsprechende Sicherungsverfügung nach Maßgabe des Kulturgüterrückgabegesetzes verbietet sich, wenn die Voraussetzungen für eine Sicherstellung nach § 8 Abs. 6 Kult GüRückG offensichtlich nicht vorliegen. (Leitsätze der Redaktion)

\section{Tenor}

- Der Bescheid des Hessischen Ministeriums für Wissenschaft und Kunst vom 15. Juli 2009 wird aufgehoben.

Die Kosten des Verfahrens werden dem Beklagten auferlegt.

Das Urteil ist hinsichtlich der außergerichtlichen Kosten des Klägers vorläufig vollstreckbar. Der Kostenschuldner darf die Vollstreckung durch Sicherheitsleistung oder Hinterlegung nach Maßgabe der Kostenfestsetzung abwenden, falls nicht der Gläubiger vor der Vollstreckung Sicherheit in derselben Höhe leistet.

\section{Tatbestand}

Die Beteiligten streiten über die Sicherstellung von 821 Münzen durch das beklagte Land. Im Zuge von Ermittlungen des Polizeipräsidiums Mittelhessen, Kriminaldirektion Gießen, gegen einen Herrn (...) wegen des Verdachts der Unterschlagung und 
der Hehlerei mit Kulturgut wurde festgestellt, dass der Kläger über das Internet Auktionshaus eBay unter dem Account „,(...) kulturhistorische Münzen erworben hatte. Alleine in der Zeit zwischen dem 13. April 2008 und dem 20. Juli 2008 wurden durch den Kläger mindestens 23 Auktionen getätigt, bei denen kulturhistorische Münzen ohne Herkunftsnachweis erworben wurden. Nach den Recherchen der Kriminaldirektion Gießen wird von eBay seit dem 1. Juli 2008 ein Herkunftsnachweis gefordert, das Einstellen ohne Expertise ist seit diesem Zeitpunkt gemäß den AGB von eBay grundsätzlich unzulässig. Gegen den Kläger wurde Strafanzeige wegen des Verdachts der Hehlerei von Kulturgut gemäß $§ 259$ StGB erstattet. Im Rahmen des Ermittlungsverfahrens wurde durch Beschluss des Amtsgerichts Wetzlar (47 Gs-2 Js 55795/08) die Durchsuchung der Wohnung des Klägers angeordnet. Am 4. Dezember 2008 wurde die Durchsuchung im Haus des Klägers durchgeführt. Laut Durchsuchungsbericht vom 4. Dezember 2008 (BI. 72/73 der Ermittlungsakte) gab der Kläger im Rahmen der Durchsuchung an, für keine seiner Münzen einen Herkunftsnachweis zu besitzen. Im Rahmen der Durchsuchung wurden 821 kulturhistorische Münzen sichergestellt. Unter dem 13. Februar 2009 verfügte die Staatsanwaltschaft Limburg - Zweigstelle Wetzlar - die Einstellung des Ermittlungsverfahrens gegen den Kläger gemäß § 170 Abs. 2 StPO. Zur Begründung wurde ausgeführt, dem Kläger sei ein vorsätzliches Handeln nicht nachweisbar, da unter anderem die „Numismatische Kommission“ der Länder der Bundesrepublik e.V. (NK) in einer gemeinsamen Erklärung vom 19. Dezember 2008 festgestellt habe, dass ein Herkunftsnachweis für die einzelne Münze nicht vorgeschrieben sei. Der Beschuldigte (der Kläger) habe daher davon ausgehen können, dass Weiterveräußerung und Erwerb von alten Münzen nicht strafbewehrt sei. Am 7. Juli 2009 verfügte die Staatsanwaltschaft Limburg sodann, die Münzen an den Kläger herauszugeben.

Vor der tatsächlichen Herausgabe der sichergestellten Münzen an den Kläger stellte das Hessische Ministerium für Wissenschaft und Kunst mit Verfügung vom 15. Juli 2009 die 821 antiken Münzen, die im Verfahren - 2 Js 55795/08 - der Staatsanwaltschaft Limburg sichergestellt wurden, gemäß $\S 40$ Nr. 2 und 4 HSOG sicher. Zur Begründung wurde ausgeführt, dass aufgrund einer gutachterlichen Beurteilung durch Herrn Dr. Noeske von der Universität Frankfurt am Main erwiesen sei, dass die weitaus meisten beschlagnahmten Münzen aus dem Ostbalkanraum (Bulgarien, Serbien) stammten. Es handele sich ausschließlich um echte, antike Fundmünzen, die nach ihrer Art, Menge und Zusammensetzung nur als Bodenfunde aus verschiedenen Grabungen stammen könnten. In allen Ländern des Ostbalkanraumes bestehe zum einen eine gesetzliche Meldepflicht für Bodenfunde an die zuständige Behörde. Zum anderen gebe es in allen Ländern Kulturgutschutzgesetze, die das Kulturgut bei seiner Entdeckung in Staatseigentum erwachsen ließen oder die Teilung des Fundeigentumes zwischen Finder und Grundstückseigentümer. Rechtmäßiger Eigentümer sei demnach entweder das Land, in dem die Münze gefunden wurde, oder (zumindest auch) der Grundstückseigentümer. Soweit Kulturgut aus dem Ausland rechtmäßig nach Deutsch- land verbracht werde, seien dafür Exportgenehmigungen der ausländischen Kulturbehörde und/oder des Zolls erforderlich. Für keine der 821 Münzen liege auch nur ein einziger der genannten möglichen Nachweise vor. Zu keiner der Münzen sei der Fundort nachgewiesen, zu keiner der Münzen sei der rechtmäßige Eigentumserwerb nachgewiesen und zu keiner der Münzen lägen Ausfuhrpapiere des Herkunftsstaates vor. Damit sei mit an Sicherheit grenzender Wahrscheinlichkeit davon auszugehen, dass die Münzen aus einem illegalen Kontext, aus Raubgrabungen, Diebstählen oder Unterschlagungen aus Museen oder Sammlungen stammten. Hierfür spreche überdies die kriminalistische und die allgemeine Lebenserfahrung. Des Weiteren sei zivilrechtlich nicht davon auszugehen, dass der Kläger rechtmäßiger Besitzer oder Eigentümer der Münzen sei. Aus diesem Grund sei davon auszugehen, dass eine Herausgabe der Münzen an den Kläger dem bisher noch nicht ermittelten Eigentümer die Wiedererlangung des Eigentums zusätzlich erschweren würde, jede weitere Veräußerung eine Hehlerei bedeute und ein wirtschaftlicher Gewinn nicht bei demjenigen erwachsen solle, der die Gegenstände lediglich unter Bruch fremder Eigentumsrechte besitze; aus diesem Grund seien die 821 Münzen gemäß $§ 40$ Nr. 2 und 4 HSOG sicherzustellen. Einer Sicherstellung nach $\S 40$ HSOG stehe auch nicht entgegen, dass die Staatsanwaltschaft zuvor das Ermittlungsverfahren gegen den Kläger eingestellt und keine Anklage erhoben habe. Eine vorherige strafprozessuale Sicherstellung schließe spätere Maßnahmen der Gefahrenabwehr nicht aus. Dass die Eigentümer der Münzen voraussichtlich nur unter großen Schwierigkeiten oder möglicherweise gar nicht mehr zu ermitteln seien, lasse die Sicherstellung des Weiteren nicht unverhältnismäßig erscheinen. Wegen der weiteren Begründung wird auf die Verfügung vom 15. Juli 2009 Bezug genommen (BI. 9 bis 12 der Gerichtsakte).

Am 31. Juli 2009 hat der Kläger Klage beim Verwaltungsgericht Wiesbaden erhoben. Zur Klagebegründung hat der Bevollmächtigte des Klägers im Wesentlichen vorgetragen, vorliegend lägen weder die Voraussetzungen des HSOG noch des Kulturgüterrückgabegesetzes vor. Insbesondere lägen die Voraussetzungen für einen Eilfall, der ein polizeiliches bzw. ordnungsbehördliches Einschreiten erforderlich gemacht hätte; nicht vor. Die 821 Münzen hätten sich durch die Beschlagnahme im Rahmen des strafrechtlichen Ermittlungsverfahrens bereits im behördlichen Gewahrsam befunden. Im Übrigen sei eine unbefristete polizeiliche Sicherung von Asservaten - um eine solche Maßnahme handele es sich beim Sicherstellungsbescheid des Beklagten - unzulässig. Auch nach der Einstellung eines Ermittlungsverfahrens richte sich die weitere Sicherstellung ausschließlich nach den Vorschriften des Strafgesetzbuches, der Strafprozessordnung und des Bürgerlichen Gesetzbuches. Schließlich lägen auch die Voraussetzungen für einen öffentlichrechtlichen Rückgabeanspruch eines UNESCO-Partnerstaates nach dem Kulturgüterrückgabegesetz nicht vor. Aus all diesen Gründen stelle die Sicherstellung der Münzen durch den Beklagten einen rechtswidrigen Eingriff in das Grundrecht des Klägers auf Eigentum dar. Wegen der weiteren Einzelheiten 
wird auf die Schreiben des Kläger-Bevollmächtigten vom 29. Juli 2009 (BI. 1-3 der Gerichtsakte) sowie vom 8. September 2009 (BI. 49/50 der Gerichtsakte) verwiesen.

\section{Der Kläger beantragt,}

den Bescheid des Hessischen Ministeriums für Wissenschaft und Kunst vom 15. Juli 2009 aufzuheben.

Der Beklagte beantragt,

die Klage abzuweisen.

Zur Klageerwiderung wird vorgetragen, das Kulturgüterrückgabegesetz enthalte keine Vollzugsbestimmungen, deswegen müsse auf das allgemeine Polizeirecht zurückgegriffen werden. Der Anwendung des $§ 40$ HSOG stehe nichts entgegen; das Ministerium für Wissenschaft und Kunst habe insoweit als besondere (Kultur-) Polizeibehörde gehandelt. Aufgrund der polizeilichen Ermittlungen sei mehr als zweifelhaft, dass der Kläger Eigentümer der beschlagnahmten Münzen sei. Wegen der weiteren Einzelheiten wird auf die Klageerwiderung vom 2. September 2009 (BI. 46/47 der Gerichtsakte) verwiesen.

Durch Beschluss vom 12. August 2009 hat sich das Verwaltungsgericht Wiesbaden für örtlich unzuständig erklärt und den Rechtsstreit an das zuständige Verwaltungsgericht Gießen verwiesen.

Durch Beschluss vom 24. Februar 2010 hat die Kammer die Entscheidung des Rechtsstreits gemäß § 6 Abs. 1 VwGO der Einzelrichterin zur Entscheidung übertragen.

Wegen der weiteren Einzelheiten des Sach- und Streitstandes wird auf den Inhalt der Gerichtsakte und der beigezogenen Behördenakten des Beklagten (2 Hefter), die Gegenstand der mündlichen Verhandlung gewesen sind, verwiesen.

\section{Entscheidungsgründe}

Die Klage ist zulässig und begründet.

Die Sicherstellungsverfügung des Hessischen Ministeriums für Wissenschaft und Kunst vom 15. Juli 2009 ist rechtswidrig und verletzt den Kläger in seinen Rechten (§ 113 Abs. 1 Satz 1 VwGO). Die Voraussetzungen für den Erlass der auf $\S 40 \mathrm{Nrn}$. 2 und 4 HSOG gestützten Sicherstellungsverfügung durch das Hessische Ministerium für Wissenschaft und Kunst vom 15. Juli 2009 lagen nicht vor.

Gemäß § 40 Nrn. 2 und 4 HSOG können die Gefahrenabwehrund die Polizeibehörden eine Sache sicherstellen, um (Nr. 2) die Eigentümerin oder den Eigentümer oder die rechtmäßige Inhaberin oder den rechtmäßigen Inhaber der tatsächlichen Gewalt vor Verlust oder Beschädigung einer Sache zu schützen, oder (Nr. 4) wenn tatsächliche Anhaltspunkte die Annahme rechtfer- tigen, dass sie zur Begehung einer Straftat oder Ordnungswidrigkeit gebraucht oder verwertet werden soll. Diese Voraussetzungen sind vorliegend nicht gegeben.

Nach Auffassung des Gerichts erweist sich die Sicherstellungsverfügung vom 15. Juli 2009 bereits als formell rechtswidrig, weil das Hessische Ministerium für Wissenschaft und Kunst die angefochtene Sicherstellungsverfügung auf der Grundlage des $\$ 40$ Nrn. 2 und 4 HSOG als unzuständige Behörde erlassen hat. Gemäß § 40 HSOG obliegt die Sicherstellung sowohl den Gefahrenabwehr- wie auch den Polizeibehörden. Nach § 1 Abs. 1 Satz 1 HSOG untergliedern sich die Gefahrenabwehrbehörden - als eine solche müsste das Hessische Ministerium für Wissenschaft und Kunst hier gehandelt haben - in Verwaltungsbehörden und Ordnungsbehörden. Gemäß $\S 2$ HSOG erfolgt die Aufgabenabgrenzung zwischen diesen Behörden dergestalt, dass die Ordnungsbehörden (allgemeine Ordnungsbehörden, Sonderordnungsbehörden) und die Polizeibehörden in Erfüllung der Aufgaben der Gefahrenabwehr außer in den Fällen des $\S 1$ Abs. 1 Satz 2 HSOG nur tätig werden, soweit die Abwehr der Gefahr durch andere Behörden, die Aufgaben der Gefahrenabwehr zu erfüllen haben, nicht oder nicht rechtzeitig möglich erscheint. Die sonstigen Aufgaben der Gefahrenabwehr erweisen sich als allgemeine Verwaltungsaufgaben, die von den Landkreisen und Gemeinden zu erfüllen sind, soweit nicht die Zuständigkeit einer Behörde der Landesverwaltung durch Rechtsvorschrift begründet ist (§ 2 Sätze 2, 3 HSOG).

Ausweislich der Ausführungen des Hessischen Ministeriums für Wissenschaft und Kunst im angefochtenen Bescheid vom 15. Juli 2009 ist das Hessische Ministerium für Wissenschaft und Kunst von seiner Zuständigkeit als Verwaltungsbehörde im Sinne des $§ 1$ Abs. 1 Satz 1 HSOG ausgegangen, die sich letztlich aus den Vorschriften des Kulturgüterrückgabegesetzes - KultGüRückG - herleitet. Gemäß § 8 Abs. 1 KultGüRückG sind die Länder - und somit aufgrund der internen Aufgabenverteilung das Hessische Ministerium für Wissenschaft und Kunst - zuständig für die erforderlichen Maßnahmen zur Ermittlung des rückgabepflichtigen Kulturgutes, seiner Sicherung und seiner Rückgabe. Die somit eröffnete generelle Zuständigkeit für die Sicherung eines rückgabepflichtigen Kulturgutes erfährt allerdings in § 8 Abs. 6 KultGüRückG eine Einschränkung dergestalt, dass das angehaltene Kulturgut nach Maßgabe der landesrechtlichen Vorschriften sicherzustellen ist, sofern zu befürchten ist, dass seine Rückgabe an den ersuchenden Staat verhindert werden soll oder dass es Schaden erleidet. Aufgrund dieser besonderen Ausformung des Eigentumsschutzes durch 8 Abs. 1, 6 KultGüRückG geht das Gericht davon aus, dass es sich bei $\S 8$ Abs. 1, 6 KultGüRückG um eine Vorschrift handelt, die als lex specialis der Vorschrift des $\S 40$ Nr. 2 HSOG vorgeht. Nur im Rahmen des $\S 8$ Abs. 1, 6 KultGüRückG war demnach eine Zuständigkeit des Hessischen Ministeriums für Wissenschaft und Kunst als Verwaltungsbehörde gemäß §§ 1 Abs. 1 Satz 1, 2 Sätze 2, 3 HSOG möglich. 
Eine Zuständigkeit des Hessischen Ministeriums für Wissenschaft und Kunst zum Erlass einer Sicherstellungsverfügung auf der Grundlage des $§ 40$ Nrn. 2 und 4 HSOG lässt sich des Weiteren nicht daraus herleiten, dass das Hessische Ministerium für Wissenschaft und Kunst vorliegend zwar nicht als Verwaltungsbehörde, sondern als zuständige Ordnungsbehörde gehandelt habe. Nach § 85 Abs. 1 Nr. 1 HSOG können die fachlich zuständigen Ministerien zwar als Landesordnungsbehörden und damit als allgemeine Ordnungsbehörden handeln; dies jedoch nur bei einem Handeln im Rahmen der fachlichen Zuständigkeit, die sich vorliegend - wie bereits ausgeführt - indes über die Vorschriften des Kulturgüterrückgabegesetzes definiert und für die Sicherstellung von Kulturgütern die Spezialregelung des $\S$ 8 Abs. 1, 6 KultGüRückG vorsieht. Eine darüber hinausgehende Zuständigkeit zur präventiven Bekämpfung von Straftaten und Ordnungswidrigkeiten durch Sicherstellungsmaßnahmen gemäß $§ 40$ Nr. 4 HSOG ist dem Hessischen Ministerium für Wissenschaft und Kunst nach Auffassung des Gerichts nicht eröffnet. Darüber hinaus zählt der Bereich des Kulturgüterschutzes nach dem Kulturgüterrückgabegesetz nicht zu den, den allgemeinen Ordnungsbehörden gemäß § 1 HSOG-DVO, § 89 Abs. 1 HSOG zugewiesenen Aufgabenbereichen.

Nach Auffassung des Gerichts erweist sich die angefochtene Verfügung vom 15. Juli 2009 auch in materieller Hinsicht als rechtswidrig. Wie bereits ausgeführt, erweist sich $\S 8$ Abs. 6 KultGüRückG gegenüber $\S 40$ Nr. 2 HSOG als lex specialis mit der Folge, dass für die Sicherstellung aus Gründen des Eigentümerschutzes nicht auf $\S 40 \mathrm{Nr}$. 2 HSOG zurückgegriffen werden kann. Vielmehr ist eine Sicherungsverfügung zum Schutze des Eigentümers nur nach Maßgabe des § 8 Abs. 6 KultGüRückG möglich. Vorliegend verbietet sich nach Auffassung des Gerichts auch eine Umdeutung der angefochtenen Verfügung vom 15. Juli 2009 in eine entsprechende Sicherungsverfügung nach Maßgabe des Kulturgüterrückgabegesetzes. Denn die Voraussetzungen für eine Sicherstellung nach $\S 8$ Abs. 6 KultGüRückG liegen offensichtlich nicht vor. Nach § 8 Abs. 6 KultGüRückG ist das angehaltene Kulturgut nach Maßgabe landesrechtlicher Vorschriften sicherzustellen, sofern zu befürchten ist, dass seine Rückgabe an den ersuchenden Staat verhindert werden soll oder dass es Schaden erleidet. Beide Voraussetzungen sind hier offensichtlich nicht gegeben. So ist nach Ausführungen der Vertreterin des Beklagten im Rahmen der mündlichen Verhandlung bislang nicht geklärt, ob und gegebenenfalls welcher Staat die Rückgabe von sichergestellten Münzen begehrt. Dass die Münzen im Besitz des Klägers Schaden erleiden würden, ist dem Gericht ebenfalls nicht ersichtlich. In diesem Zusammenhang verweist das Gericht vielmehr darauf, dass es nach der Konzeption des § 8 KultGüRückG den Regelfall darstellt, dass das angehaltene Kulturgut zunächst bei seinem Besitzer verbleibt, der aber wegen der Anhaltung - sofern eine solche nach Abs. 3 des $\S 8$ KultGüRückG ausgesprochen wurde, was vorliegend indes bislang wohl nicht geschehen ist - mit dem Verbot, das betroffene Kulturgut an andere Personen weiterzugeben, belegt ist.
Aber auch die Voraussetzungen für eine Sicherstellung der beschlagnahmten Münzen nach § 40 Nr. 4 HSOG sind nach Auffassung des Gerichts nicht gegeben. Eine Sicherstellung nach $\S 40 \mathrm{Nr}$. 4 setzt voraus, dass tatsächliche Anhaltspunkte die Annahme rechtfertigen, dass die sichergestellte Sache zur Begehung einer Straftat oder Ordnungswidrigkeit gebraucht oder verwertet werden soll. Dies setzt einen hinreichenden entsprechenden Verdacht voraus, der nicht auf bloßen Vermutungen, sondern auf Tatsachen beruhen muss. Anhaltspunkte hierfür sind nach Auffassung des Gerichts nicht gegeben und können insbesondere auch nicht daraus hergeleitet werden, dass es sich beim Kläger um einen Münzsammler handelt und es unter Münzsammlern gebräuchlich ist, dass Münzen gekauft und verkauft werden.

Da die angefochtene Verfügung die Besitzrechte des Klägers an den sichergestellten Münzen verletzt, war die Sicherstellungsverfügung vom 15. Juli 2009 aufzuheben.

Die Kosten des Verfahrens hat der Beklagte zu tragen, da er unterlegen ist ( $\$ 154$ Abs. 1 VwGO).

Der Ausspruch über die vorläufige Vollstreckbarkeit und Abwendungsbefugnis beruht auf $\S 167 \mathrm{VwGO}$ iVm $\S § 708$ Nr. 11, $711 \mathrm{ZPO}$.

Die Berufung war nicht zuzulassen, da die Rechtssache nach Auffassung des Gerichts wegen der fehlenden Zuständigkeit des Beklagten keine grundsätzliche Bedeutung aufweist ( $\S \S$ 124a Abs. 1 Satz 1, 124 Abs. 2 Nr. 3 VwGO).

\section{Beschluss}

Der Streitwert wird auf 20.000 Euro festgesetzt. (...) Gemäß $\S 52$ Abs. 3 GKG wurde der Wert der beschlagnahmten Münzen bei der Streitwertbemessung in Ansatz gebracht. (Entscheidung von der Redaktion bearbeitet.) 